\title{
Energy-Efficient Clustering Scheme in Wireless Sensor Network
}

\author{
Joong-Ho Lee \\ Dept. of Computer Science, Yongin University, Korea \\ Joongho65@yongin.ac.kr
}

\begin{abstract}
Hierarchical clustering algorithms have been proposed to minimize energy consumption by sensor nodes under power constraints in a wireless sensor network. However, previously reported methods such as backbone construction algorithms consume energy quickly at the cluster head nodes. A cluster head node must therefore be changed periodically by election among the available sensor nodes, to transfer aggregated data from one cluster head node to another for as long as possible. This study investigates the clustering algorithm and cluster head election methodology among sensors to efficiently control energy consumption. This paper presents comparison results between the conventional and proposed schemes of clustering algorithms.
\end{abstract}

Keywords: hierarchical clustering algorithm, wireless sensor network, cluster head node, sensor node, energy consumption

\section{Introduction}

Wireless sensor networks (WSNs) have witnessed significant progress over the last few decades. This has led to development of a wide range of applications. Owing to the development of semiconductor technology, micro-electro-mechanical-system (MEMS) sensor design technology has achieved low-power operation and enabled development of relatively inexpensive wireless micro-sensors that can be widely applied to WSNs. [1] Sensors in a WSN, can monitor the natural environment in large areas under harsh weather conditions and limitations of power constraints. Data aggregation and transmission by these sensors must ensure robust operation under power constraints, even when exposed to hostile environments $[2,3]$.

Tiny sensors can be used in high-quality network applications requiring hundreds or thousands of sensors. It is almost impossible to replace batteries in such large-scale deployed networks because there is no human intervention after deployment. [4] Energyefficient routing to save the battery power of sensor nodes is a key issue in a WSN, which consists of a base station and hundreds or thousands of sensor nodes. The grouping algorithm of the sensor nodes has been widely researched in the field of communication to minimize power consumption. [5] Conventional clustering technology builds clusters based on a uniform network environment. However, in a real environment, a non-uniform network can be formed depending on the geographical characteristics or the sensor node distribution method. [6] In this non-uniform network environment, when the existing absolute hop-distance-based clustering technique is applied, clusters in a region with higher sensor node density have larger number of sensor nodes than clusters in regions with a relatively low node density. Therefore, such an environment would result in uneven clusters. Cluster head $(\mathrm{CH})$ nodes in uneven clusters increase energy consumption, which causes unbalanced energy consumption in the sensor network, thereby shortening network lifetime. [7] When a CH node is powered off, it cannot send data packets to other nodes. Therefore, one of the other head nodes should act as the new cluster node for

Received (June 15, 2018), Review Result (September 28, 2018), Accepted (October 10, 2018) 
transmission of data packets. This reduces the overall network lifetime. [8, 9] It is also necessary to consider $\mathrm{CH}$ node lifetime when forming a cluster to maintain longer network lifetime. Energy-efficient routings for WSNs such as low energy adaptive clustering hierarchy (LEACH) scheme [10] use single-hop clustering algorithms in which the $\mathrm{CH}$ node communicates with a sink node directly. However, this algorithm is inefficient for a $\mathrm{CH}$ node that is located at a distance greater than one hop from the sink node.

Therefore, in this study, we propose a method to construct evenly sized clusters in an environment with WSNs, where sensor nodes are deployed non-uniformly to improve the network lifetime. This paper presents a clustering algorithm based on distances between sensor nodes, which enables quickly and power-efficient constructions of sensor groups. The algorithm for election of the $\mathrm{CH}$ node is also presented. Further, comparison results between the proposed and conventional algorithms are presented based on the results from the simulations.

\section{Related Review}

$\mathrm{CH}$ nodes consume more energy than member nodes because they have to receive data from member nodes. However, they are efficient for power saving compared to direct communication with sink node. [11] The LEACH scheme has been proposed to solve this problem of energy consumption. [12] LEACH is a single-hop clustering scheme for constructing clusters having a plurality of locally adjacent nodes as members, merging the collected data, and transmitting them to the sink node. LEACH periodically selects a $\mathrm{CH}$ node within a cluster as an arbitrary node. The selected $\mathrm{CH}$ node transmits a message to other nodes within the communication area, and the node that receives the message consists of members of the $\mathrm{CH}$ node. In this manner, a cluster group is formed. A sensor node directly transmits data to the $\mathrm{CH}$ node, which is located in a cluster group area. $\mathrm{LEACH}$ is a way to extend network lifetime by periodically replacing $\mathrm{CH}$ nodes. It is energy efficient compared to direct transmission to the sink node between all sensor nodes.

\subsection{Radio Channel Model}

LEACH algorithm uses a simplified radio model $E_{\text {device }}=50 \mathrm{~nJ} / \mathrm{bit}$ to operate transmitter/receiver devices, with $\epsilon_{a m p}=100 \mathrm{pJ} / \mathrm{bit} / \mathrm{m}^{2}$. [12] Figure 1 shows the radio model.

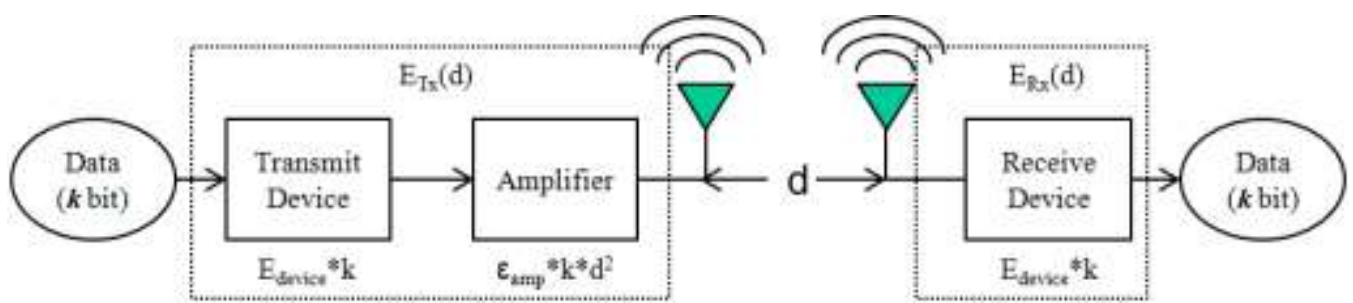

Figure 1. Radio Energy Model Between Transmit and Receive Nodes

To transmit a k-bit message to a distance $\mathrm{d}$, the radio model expands as follows:

$$
\begin{aligned}
& E_{T x}(k, d)=E_{T x-\text { device }}(k)+E_{T x-a m p}(k, d) \\
& E_{T x}(k, d)=E_{\text {device }} * k+\epsilon_{\text {amp }} * k * d^{2}
\end{aligned}
$$

To receive a k-bit message from the transmit node, the radio model expands as follows [12]: 


$$
\begin{aligned}
& E_{R x}(k, d)=E_{R x-\text { device }}(k) \\
& E_{R x}(k, d)=E_{\text {device }} * k
\end{aligned}
$$

Based on previous assumptions, it is seen that total wireless energy consumption depends on the routing method. If the routing method passes through $n$ paths, the total energy consumption is as follows:

$$
\begin{aligned}
& \left(E_{T x}(k, n * d)+E_{R x}(k, n * d)\right)= \\
& \left(E_{\text {device }} * k+\epsilon_{\text {amp }} * k *(n * d)^{2}+E_{\text {device }} * k\right)
\end{aligned}
$$

The distance between nodes on each path is assumed to be the same, i.e., d.

\section{Cluster Formation}

This paper presents a clustering algorithm based on distance. The concept of cluster formation is based on the principle of reciprocal attraction between nodes. For cluster formation based on adjacent nodes, power of the sensor node between adjacent nodes is defined as $f_{A B}$ (from node $A$ to $B$ ) according to the physical distance, and is shown in Figures 2-a and 2-b. To model the interaction forces between nodes, the distance between nodes is expressed as unit distance on the $\mathrm{x}$ and $\mathrm{y}$ axes. The distance between two nodes $(A-B)$ is $d l$, which is the same as in case 1 , and the interaction force is expressed in inverse proportion to the distance. Mutual attraction force from node A to B is defined as$f_{A B}$. If we expand the number of adjacent nodes to 3 , then two types of cases exist between the nodes: the case 2 and 3. From this, the interaction force can be calculated based on the distance between each node. Case 4-7 constitute a model that calculates the interaction with the number of cases that can be formed when the number of adjacent nodes is four. Similarly, the number of adjacent nodes can be expanded, and the interaction force for each type can be calculated. When mutual node forces $f_{A B}$ and $f_{A C}$ are orthogonal to each other, the mutual attraction given by $f_{B C}$ is calculated as in case 3 . Therefore, the mutual attractive force between $f_{A B}$ and $f_{A C}$ is greater than- $f_{B C}$. If $f_{A B}$ and $f_{A C}$ are not orthogonal, then the distance between nodes $B$ and $C$ in case 3 should be expressed differently. Therefore, the magnitude of the mutual force also changes. Based on the modeling of the distance between sensor nodes in Figure 2, groups are formed between adjacent sensor nodes. Figure 3 shows the process of group formation for arbitrarily deployed sensor nodes. The neighboring nodes have diagrammed the process of gathering nodes located close to each other. For nodes in coordinates $(4,4)$, the pulling forces on both sides have similar strength however, they eventually belong to the strong pulling group. Consequently, three groups are formed and nodes located at coordinates (4, 4) are registered as members of group B. In this way, applying interaction force between nodes and to adjacent nodes makes it possible to form a cluster. Figure 4 shows an example in which approximately 1000 sensors are randomly distributed within a $100 \times 100$ unit area, and then, clusters are formed between adjacent nodes. Figure 4 shows simulation results of the proposed clustering algorithm. The cluster formation consists of two steps. First, in the randomly scattered sensor nodes, the nodes search the distance1(1d) node between each node. If they find distance- 1 node, they communicate with each other and note the id number of each node for identification. Second, each node searches for adjacent nodes that extend to distance-2(2d). In this study, the distance between nodes affected by reciprocal attraction is limited to $2 \mathrm{~d}$. 


\begin{tabular}{|c|c|c|}
\hline & Case & force (node A to node B) : faB \\
\hline \multirow{3}{*}{1} & \multirow{3}{*}{ 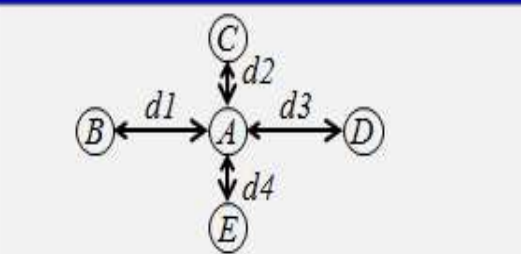 } & $f_{A B}=1 / d 1, f_{A C}=1 / d 2, f_{A D}=1 / d 3, f_{A E}=1 / d 4$ \\
\hline & & $f_{B C}=1 /\left(\sqrt{d 1^{2}+d 2^{2}}\right), f_{B E}=1 /\left(\sqrt{d 1^{2}+d 4^{2}}\right)$ \\
\hline & & $f_{C D}=1 /\left(\sqrt{d 2^{2}+d 3^{2}}\right), f_{D E}=1 /\left(\sqrt{d 3^{2}+d 4^{2}}\right)$ \\
\hline \multirow{3}{*}{2} & \multirow{3}{*}{ 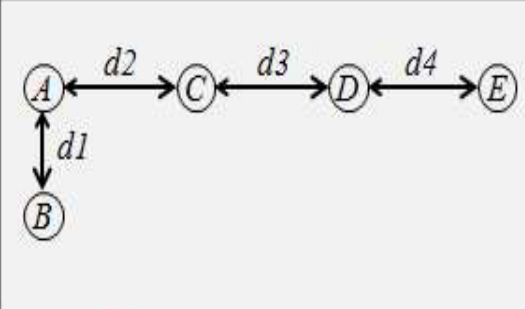 } & $f_{A B}=1 / d 1, f_{A C}=1 / d 2, f_{C D}=1 / d 3, f_{D E}=1 / d 4$ \\
\hline & & $\begin{array}{l}f_{A D}=1 /(d 2+d 3), f_{C E}=1 /(d 3+d 4) \\
f_{A B}=1 /(d 2+d 3+d 4)\end{array}$ \\
\hline & & $\begin{array}{l}f_{B C}=1 /\left(\sqrt{d 1^{2}+d 2^{2}}\right), f_{B D}=1 /\left(\sqrt{d 1^{2}+(d 2+d 3)^{2}}\right. \\
f_{B E}=1 /\left(\sqrt{d 1^{2}+(d 2+d 3+d 4)^{2}}\right.\end{array}$ \\
\hline \multirow[b]{2}{*}{3} & \multirow{2}{*}{ 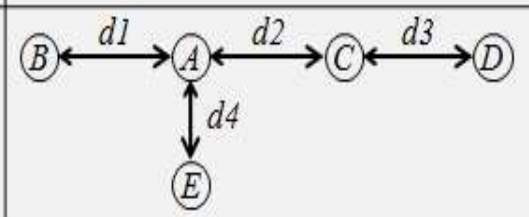 } & $f_{A B}=1 / d 1, f_{A C}=1 / d 2, f_{C D}=1 / d 3, f_{A D}=1 / d 4$ \\
\hline & & $\begin{array}{l}f_{B E}=1 /\left(\sqrt{d 1^{2}+d 4^{2}}\right), f_{C E}=1 /\left(\sqrt{d 2^{2}+d 4^{2}}\right), \\
f_{D E}=1 /\left(\sqrt{d 4^{2}+(d 2+d 3)^{2}}\right.\end{array}$ \\
\hline \multirow[b]{3}{*}{4} & \multirow{3}{*}{$\begin{array}{l}\text { (A) } \stackrel{d 1}{\longleftrightarrow} \text { (B) } \stackrel{d 2}{\longleftrightarrow} \text { (C) } \\
\downarrow d 3 \\
(D) \\
\downarrow d 4 \\
\text { (E) }\end{array}$} & $f_{A B}=1 / d 1, f_{B C}=1 / d 2, f_{A D}=1 / d 3, f_{D E}=1 / d 4$ \\
\hline & & $f_{A C}=1 /(d l+d 2), f_{A E}=1 /(d 3+d 4)$ \\
\hline & & $\begin{array}{l}f_{B D}=1 /\left(\sqrt{d 1^{2}+d 3^{2}}\right), f_{C D}=1 /\left(\sqrt{d 3^{2}+(d 1+d 2)^{2}},\right. \\
f_{B E}=1 /\left(\sqrt{d 1^{2}+(d 3+d 4)^{2}},\right. \\
f_{C E}=1 /\left(\sqrt{(d 1+d 2)^{2}+(d 3+d 4)^{2}}\right.\end{array}$ \\
\hline \multirow{3}{*}{5} & \multirow{3}{*}{$(A) \stackrel{d 1}{\longleftrightarrow}(B) \stackrel{d 2}{\longleftrightarrow}(C) \stackrel{d 3}{\longleftrightarrow}(D) \stackrel{d 4}{\longleftrightarrow}$ (E) } & $f_{A B}=1 / d 1, f_{B C}=1 / d 2, f_{C D}=1 / d 3, f_{D E}=1 / d 4$ \\
\hline & & $f_{A C}=1 /(d 1+d 2), f_{B D}=1 /(d 2+d 3), f_{C E}=1 /(d 3+d 4)$ \\
\hline & & $\begin{array}{l}f_{A D}=1 /(d l+d 2+d 3), f_{B E}=1 /(d 2+d 3+d 4) \\
f_{A E}=1 /(d l+d 2+d 3+d 4)\end{array}$ \\
\hline \multirow{4}{*}{6} & \multirow{4}{*}{ 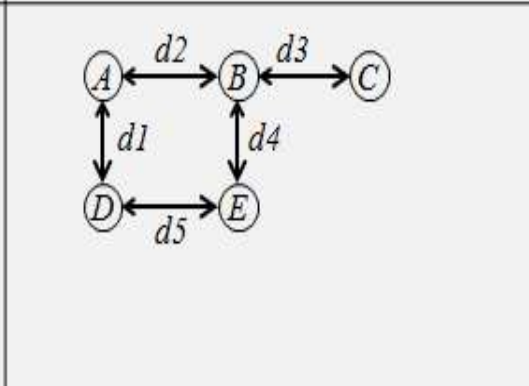 } & $f_{A B}=1 / d 2, f_{B C}=1 / d 3, f_{A D}=1 / d 1, f_{B E}=1 / d 4, f_{B C}=1 / d 3$ \\
\hline & & $f_{A C}=1 /(d 2+d 3)$ \\
\hline & & $\begin{array}{l}f_{A E}=1 /\left(\sqrt{d 2^{2}+d 4^{2}}\right)=1 /\left(\sqrt{d 1^{2}+d 5^{2}}\right), \\
f_{B D}=1 /\left(\sqrt{d 1^{2}+d 2^{2}}\right)=1 /\left(\sqrt{d 4^{2}+d 5^{2}}\right), \\
f_{C E}=1 /\left(\sqrt{d 3^{2}+d 4^{2}}\right)\end{array}$ \\
\hline & & $f_{C D}=1 /\left(\sqrt{d 1^{2}+(d 2+d 3)^{2}}\right.$ \\
\hline
\end{tabular}

Figure 2-a. Distance between Sensor Nodes vs. Force Modeling 


\begin{tabular}{|c|c|c|}
\hline & Case & force (node A to node B) : fAB \\
\hline \multirow{3}{*}{1} & \multirow{3}{*}{ 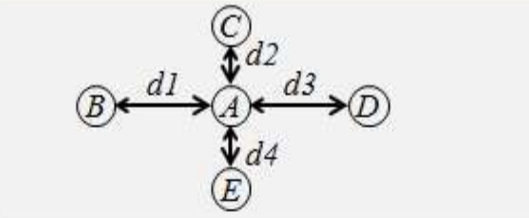 } & $f_{A B}=1 / d 1, f_{A C}=1 / d 2, f_{A D}=1 / d 3, f_{A E}=1 / d 4$ \\
\hline & & $f_{B C}=1 /\left(\sqrt{d 1^{2}+d 2^{2}}\right), f_{B E}=1 /\left(\sqrt{d 1^{2}+d 4^{2}}\right)$ \\
\hline & & $f_{C D}=1 /\left(\sqrt{d 2^{2}+d 3^{2}}\right), f_{D E}=1 /\left(\sqrt{d 3^{2}+d 4^{2}}\right)$ \\
\hline \multirow{3}{*}{2} & \multirow{3}{*}{$\underset{\coprod_{d} d 1}{\stackrel{d 2}{\longleftrightarrow}}(C) \stackrel{d 3}{\longleftrightarrow}(D) \stackrel{d 4}{\longleftrightarrow}(E)$} & $f_{A B}=1 / d 1, f_{A C}=1 / d 2, f_{C D}=1 / d 3, f_{D E}=1 / d 4$ \\
\hline & & $\begin{array}{l}f_{A D}=1 /(d 2+d 3), f_{C E}=1 /(d 3+d 4), \\
f_{A E}=1 /(d 2+d 3+d 4)\end{array}$ \\
\hline & & $\begin{array}{l}f_{B C}=1 /\left(\sqrt{d 1^{2}+d 2^{2}}\right), f_{B D}=1 /\left(\sqrt{d 1^{2}+(d 2+d 3)^{2}}\right. \\
f_{B E}=1 /\left(\sqrt{d 1^{2}+(d 2+d 3+d 4)^{2}}\right.\end{array}$ \\
\hline \multirow[b]{2}{*}{3} & \multirow{2}{*}{ 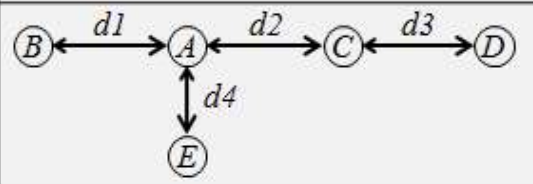 } & $f_{A B}=1 / d 1, f_{A C}=1 / d 2, f_{C D}=1 / d 3, f_{A D}=1 / d 4$ \\
\hline & & $\begin{array}{l}f_{B E}=1 /\left(\sqrt{d 1^{2}+d 4^{2}}\right), f_{C E}=1 /\left(\sqrt{d 2^{2}+d 4^{2}}\right), \\
f_{D E}=1 /\left(\sqrt{d 4^{2}+(d 2+d 3)^{2}}\right.\end{array}$ \\
\hline \multirow[b]{3}{*}{4} & \multirow{3}{*}{ (A) $\stackrel{d 1}{\longleftrightarrow}(B) \stackrel{d 2}{\longleftrightarrow}$ (C) } & $f_{A B}=1 / d 1, f_{B C}=1 / d 2, f_{A D}=1 / d 3, f_{D E}=1 / d 4$ \\
\hline & & $f_{A C}=1 /(d 1+d 2), f_{A E}=1 /(d 3+d 4)$ \\
\hline & & $\begin{array}{l}f_{B D}=1 /\left(\sqrt{d 1^{2}+d 3^{2}}\right), f_{C D}=1 /\left(\sqrt{d 3^{2}+(d 1+d 2)^{2}},\right. \\
f_{B E}=1 /\left(\sqrt{d 1^{2}+(d 3+d 4)^{2}},\right. \\
f_{C E}=1 /\left(\sqrt{(d 1+d 2)^{2}+(d 3+d 4)^{2}}\right.\end{array}$ \\
\hline \multirow{3}{*}{5} & \multirow{3}{*}{ (A) $\stackrel{d 1}{\longleftrightarrow}(B) \stackrel{d 2}{\longleftrightarrow}(C) \stackrel{d 3}{\longleftrightarrow}(D) \stackrel{d 4}{\longleftrightarrow}$ (E) } & $f_{A B}=1 / d 1, f_{B C}=1 / d 2, f_{C D}=1 / d 3, f_{D E}=1 / d 4$ \\
\hline & & $f_{A C}=1 /(d 1+d 2), f_{B D}=1 /(d 2+d 3), f_{C E}=1 /(d 3+d 4)$ \\
\hline & & $\begin{array}{l}f_{A D}=1 /(d 1+d 2+d 3), f_{B E}=1 /(d 2+d 3+d 4) \\
f_{A E}=1 /(d 1+d 2+d 3+d 4)\end{array}$ \\
\hline \multirow{4}{*}{6} & \multirow{4}{*}{ (A) $\stackrel{d 2}{\longleftrightarrow}(B) \stackrel{d 3}{\longleftrightarrow}$ (C) } & $f_{A B}=1 / d 2, f_{B C}=1 / d 3, f_{A D}=1 / d 1, f_{B E}=1 / d 4, f_{B C}=1 / d 3$ \\
\hline & & $f_{A C}=1 /(d 2+d 3)$ \\
\hline & & $\begin{array}{l}f_{A E}=1 /\left(\sqrt{d 2^{2}+d 4^{2}}\right)=1 /\left(\sqrt{d 1^{2}+d 5^{2}}\right), \\
f_{B D}=1 /\left(\sqrt{d 1^{2}+d 2^{2}}\right)=1 /\left(\sqrt{d 4^{2}+d 5^{2}}\right), \\
f_{C E}=1 /\left(\sqrt{d 3^{2}+d 4^{2}}\right)\end{array}$ \\
\hline & & $f_{C D}=1 /\left(\sqrt{d 1^{2}+(d 2+d 3)^{2}}\right.$ \\
\hline
\end{tabular}

Figure 2-b. Distance between Sensor Nodes vs. Force Modeling

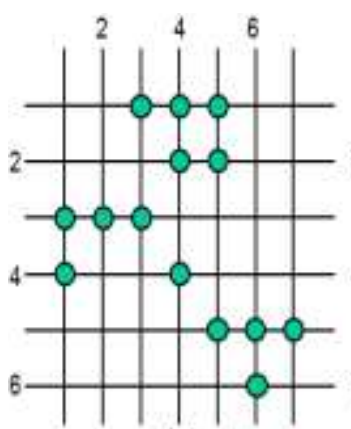

(a)

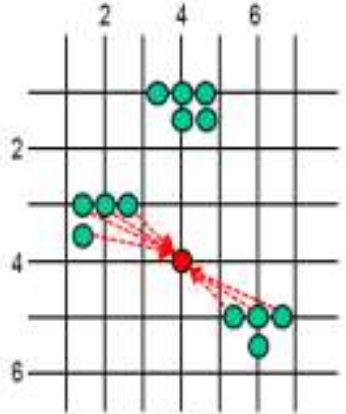

(b)

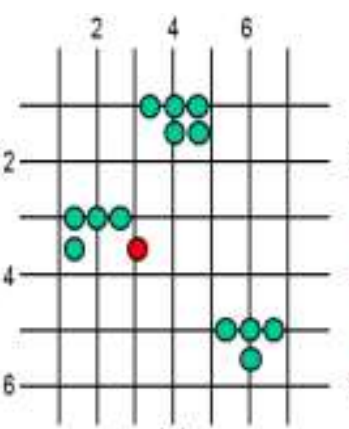

(c)

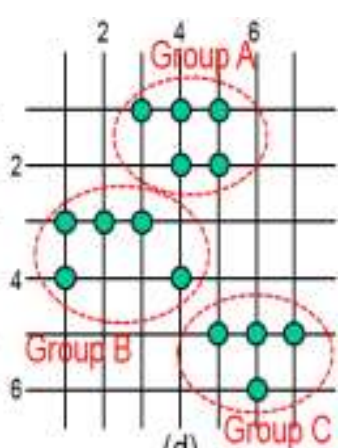

(d)

Figure 3. Example of Group Formation 


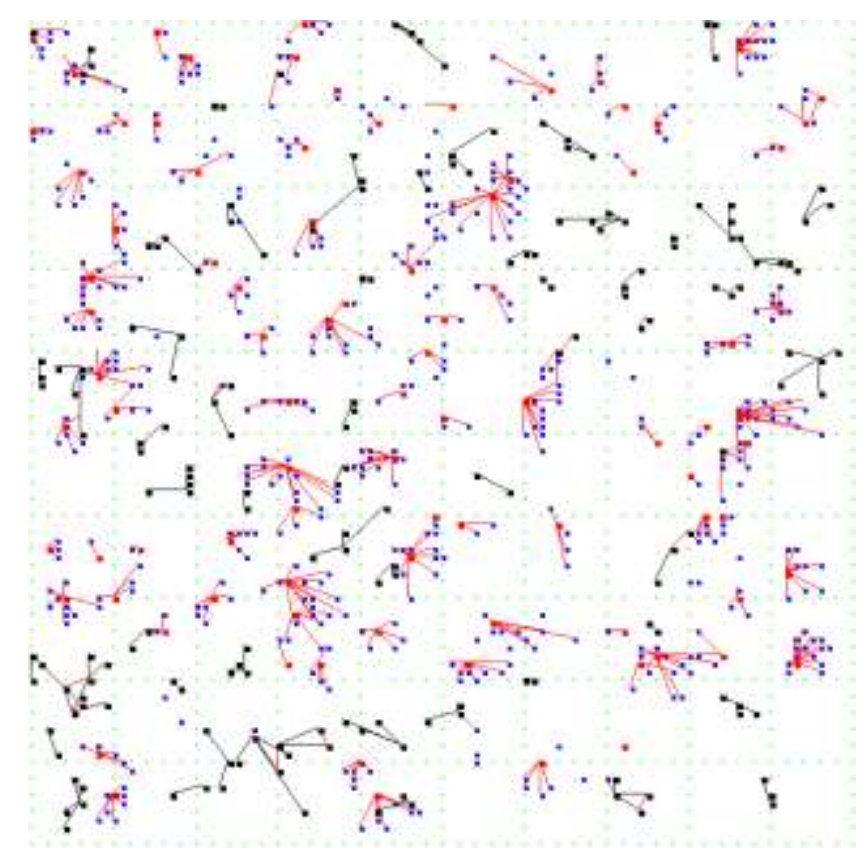

Figure 4. Sensor Node Clustering Simulation for a 100×100 Unit Area

\subsection{Clustering}

The following exaggeration is performed to form a cluster group between adjacent nodes in the distance model based on the distance of adjacent nodes shown in Figures 2-a and 2-b.

p1. All nodes send broadcast message including coordinate and distance information to neighboring nodes.

p2. A node receives a broadcast message including coordinate and distance information from the adjacent node. Calculate distance from the adjacent node from which message is received.

p3. Send group member request message.

p4. Receive an acknowledgment message from the requested node.

p5. Each node registers the node that received the acknowledgment message as its own member

Group formation for all the sensor nodes is completed by performing the above processes $\mathrm{p} 1-\mathrm{p} 5$.

\subsection{Cluster Head Election}

After cluster formation, each cluster group has to elect a $\mathrm{CH}$ node. Each node has connectivity to the nodes among the members of the cluster group. From this connectivity, the node having maximum connectivity among the members can be found. This node is selected to be a $\mathrm{CH}$ node. The process of selecting the $\mathrm{CH}$ node from the formed cluster is shown below.

p6. Calculate connectivity from member nodes of a group

p7. Remove the least connected node first

p8. Return to p6 until there are no adjacent nodes

p9. Broadcast the $\mathrm{CH}$ node to the members

p10. All member nodes register the $\mathrm{CH}$.

Selection of the $\mathrm{CH}$ node is completed by the procedure shown by p6-p10. Figure 5 shows the election process of the $\mathrm{CH}$ node from members belonging to a sample cluster. 
The process of excluding cluster members one at a time from steps (a) to (i) is shown for a certain cluster group, and a member node corresponding to the coordinates $(3,4)$ is selected as a $\mathrm{CH}$ node.

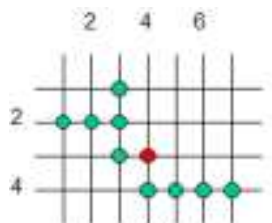

(a)

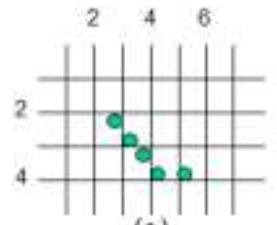

(e)

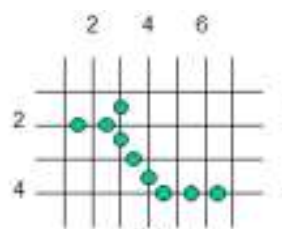

(b)

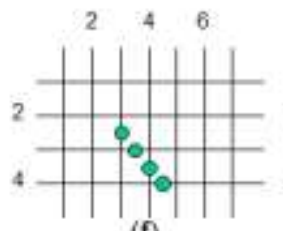

(f)

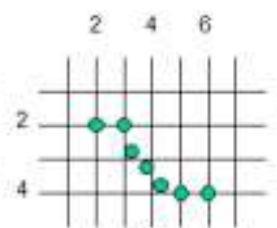

(c)

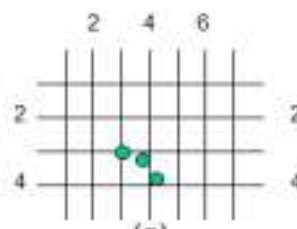

(g)

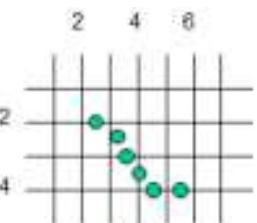

(d)

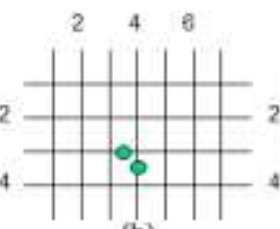

(h)

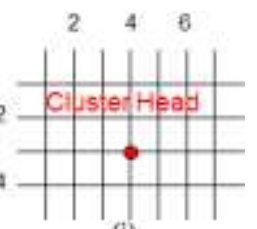

(i)

Figure 5. Example of $\mathrm{CH}$ Node Election

\subsection{Consideration on Energy Consumption Due to Routing}

Shortest path from the source to the sink node in the routing algorithm may be inefficient for energy consumption. Energy consumption of the source node increases quickly when the direct communication method is applied from the source to the sink node. Owing to these problems, routing schemes are applied to reduce energy consumption. However, when the source node is close to the sink node, the direct communication method is better in terms of energy efficiency. Figure 6 shows results of the two cases by simulating the number of nodes passing through the distance-based shortest path and through the round-trip at a specific node. As a result of simulation, the number of nodes that pass through the shortest path is larger than that of the bypass path. Results of the simulation with 100 times accumulation are shown in Figure 7. Adjacent paths are close in distance; therefore, there is room for improvement in energy efficiency by reducing the number of paths to improve energy efficiency.

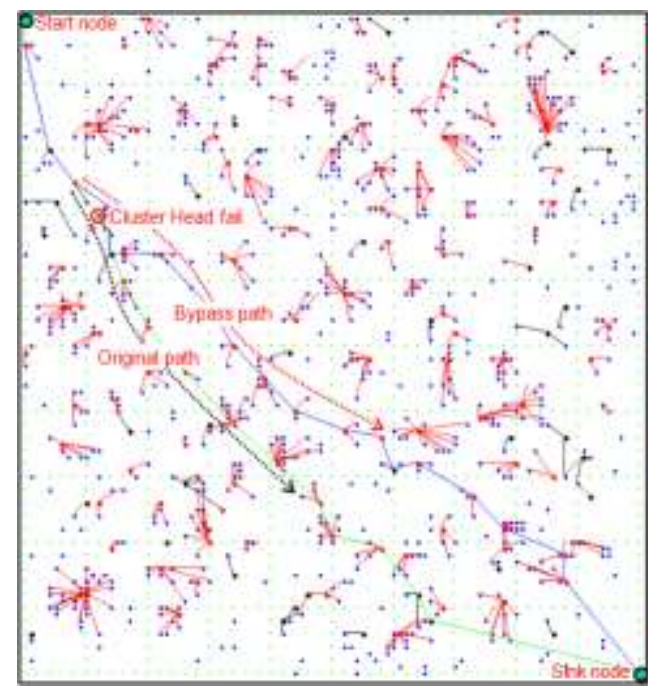

(a) Simulation example 1

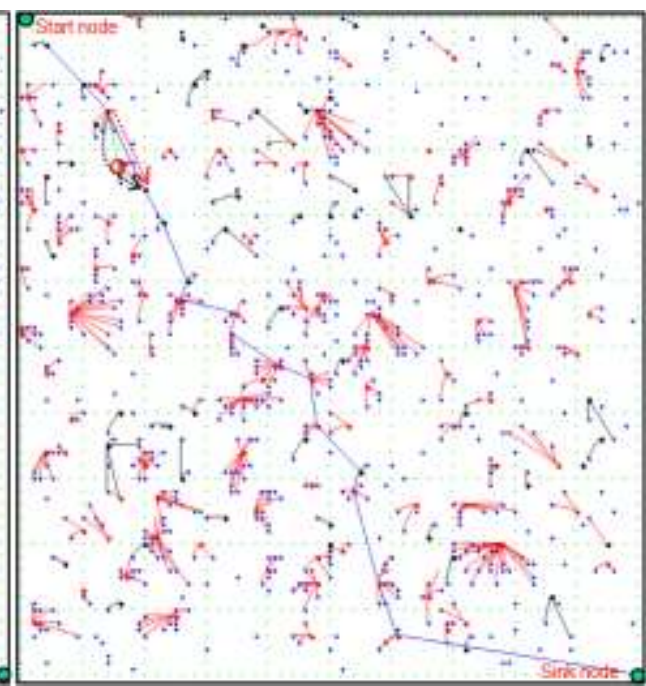

(b) Simulation example 2

Figure 6. Routing Path Simulation between Short and Bypass Path 


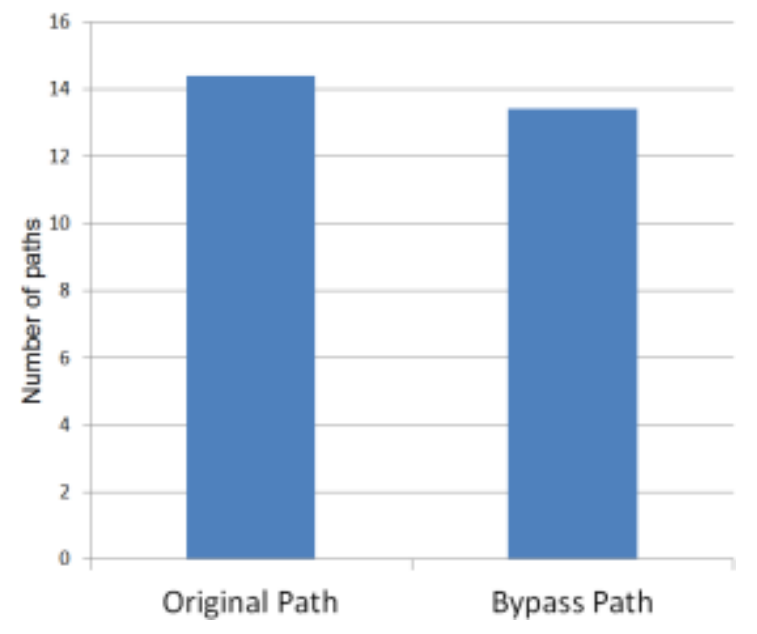

Figure 7. Number of Paths between Short and Bypass Path

\subsection{Cluster Uniformity}

Even in an environment where sensor nodes are deployed randomly, it is necessary to construct a uniform cluster to improve the lifetime of the network. In this study, we propose a second clustering process based on the clusters formed in the first stage for cluster homogenization. From the simulation results of the first stage (Figure 8 (a)), it is seen that the ungrouped sensor nodes still exist. By executing the second step of clustering, it can be confirmed that the clusters are formed more uniformly in the group where clusters are not formed or cluster members are less in the first step. The proposed method uses multi-hop-shortest distance based clustering method and selects member nodes so that clustering based on Figure 8 (b) and the shortest distance hop count can be performed.

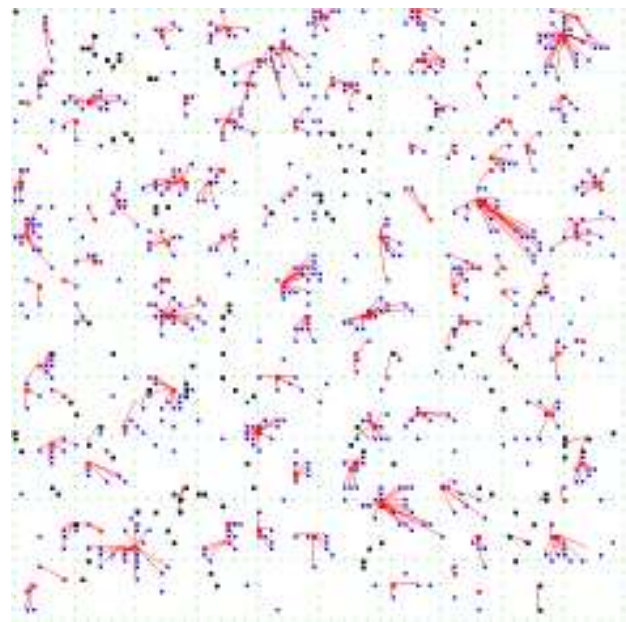

(a) Clustering First Step

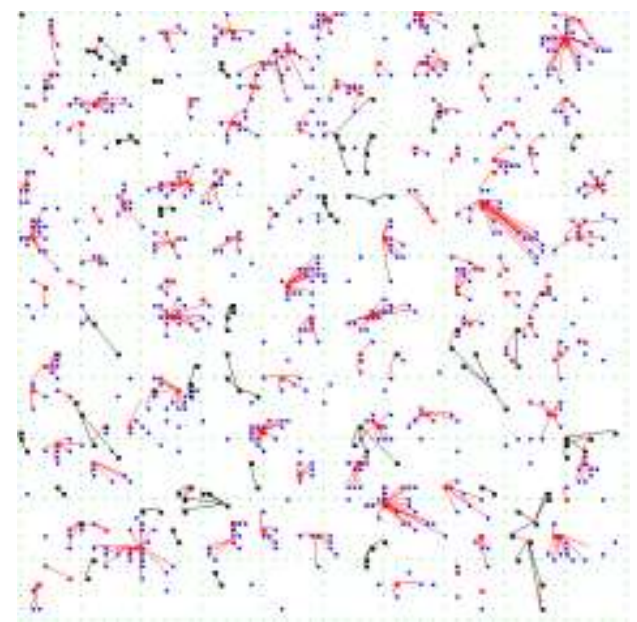

(b) Clustering Second Step

Figure 8. Cluster Uniformization Process

\section{Simulation Results}

Verification of the size of members per group for the results of the cluster group is needed. Figure 9 shows the cluster group distribution vs. cluster members. If the number of members per group is considerably large, it is necessary to divide the group into two or three sub-groups. 


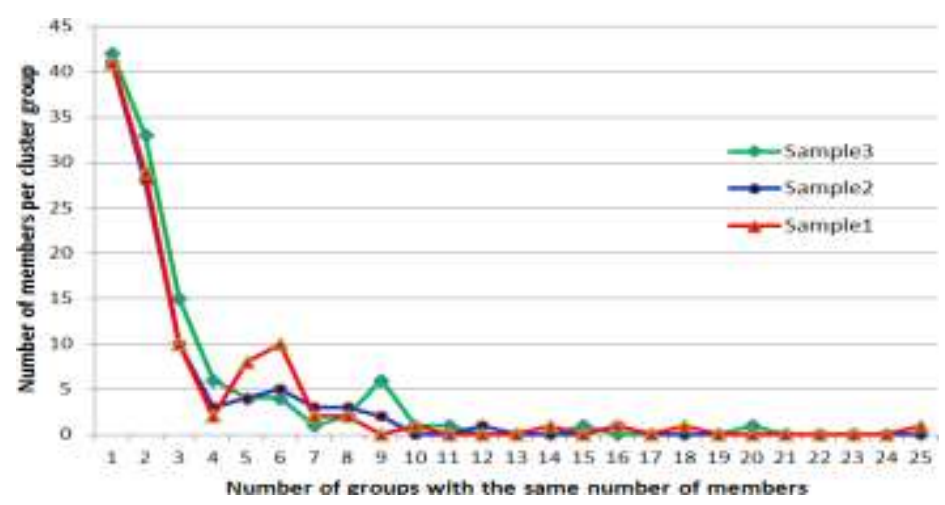

\section{Figure 9. Simulation Results for Number of Groups with the Same Number of Members}

Figure 10 shows the degree of uniformization after the cluster uniformization process. Simulation results are accumulated 100 times for approximately 1000 nodes in a $100 \times 100$ unit area. The $\mathrm{x}$-axis represents a cluster group with the same number of members, and the $y$-axis represents the number of cluster groups of the same member. In other words, the number of groups having one cluster member is $\sim 60$ in the first step, whereas the number of groups after the second step is reduced to $\sim 40$ after the cluster homogenization process. On the contrary, in the first step, the number of groups having 6 cluster members is $\sim 10$, and after the second step, it is increased to $\sim 20$ after the process of homogenizing the cluster. If the number of members per group is large, it is necessary to divide the group into two or three sub-groups. Consequently, the number of groups with more than five members in the cluster group increased, and the number of cluster groups with fewer than five members decreased, thereby improving the uniformity as a whole. To further improve the cluster equalization rate, several additional steps can be taken, as shown in Figure 8.

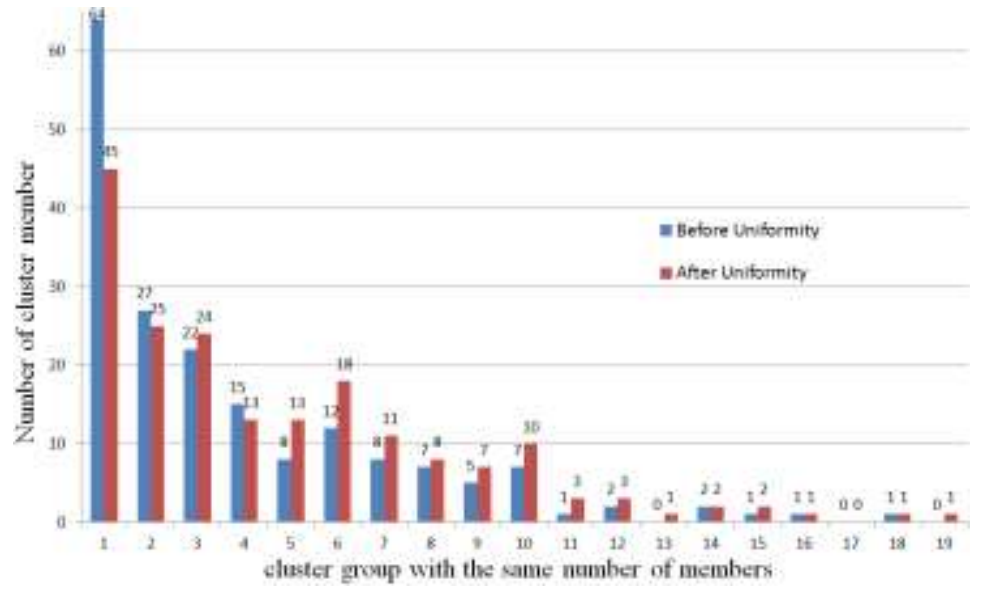

Figure 10. Degree of Uniformity According to the Cluster Group Forming Step

\section{Conclusion}

This paper describes a clustering algorithm based on distance and shows the results of cluster formation in Figure 4. It also shows the algorithm of $\mathrm{CH}$ node election. Proposed algorithm is based on the concept of reciprocal attraction between nodes. We started with the assumption that there is a pulling force (reciprocal attraction) between nodes. To improve cluster uniformization, we proposed an additional clustering process based on distance. As shown in Figure 8, 
we obtained improved results for the uniformity of the clusters. The degree of uniformity can be improved by repeating the clustering step. The proposed clustering algorithm is effective for clustering of WSNs because it is simple to implement and is expected to be effective for low power implementation.

\section{Acknowledgments}

This paper is a revised and expanded version of a paper entitled "Scheme of Clustering in a Wireless Sensor Network", presented at 7th International Conference on Next Generation Computer and Information Technology (NGCIT 2018), Royton Sapporo, Hokkaido, Japan last August 23 - 25, 2018.

\section{References}

[1] Md. Zair Hussain, M. P. Singh and R. K. "Singh Analysis of Lifetime of Wireless Sensor Network", International Journal of Advanced Science and Technology, vol. 53, (2013) April, pp. 117-126.

[2] J. Hill Jason Hill, R. Szewczyk, A. Woo, S. Hollar, D. Culler and K. Pister, "System Architecture Directions for Networked Sensors", Proc. Ninth Int'l Conf. Architectural Support for Programming Languages and Operating Systems (ASPLOS '00), (2000), pp. 93-104.

[3] S. Mukhopadhyay, D. Panigrahi and S. Dey, "Model Based Error Correction for Wireless Sensor Networks", Sensor and Ad Hoc Communications and Networks, (2004) October 4-7.

[4] L. Jianzhong and G. Hong, "Survey on sensor network research", Journal of Computer Research and Development, vol. 45, no. 1, (2008), pp. 1-15.

[5] C. Liu, K. Wu and J. Pei, "An Energy-Efficient Data Collection Framework for Wireless Sensor Networks by Exploiting Spatiotemporal Correlation", IEEE Transaction on Parallel and Distributed Systems, vol. 18, (2007) July, pp. 1010-1023.

[6] S. Wong, J. Lim, S. Rao and W. Seah, "Multihop Localization with Density and Path Length Awareness in Non-Uniform Wireless Sensor Networks", Proc. Of the International Symposium on Parallel Architectures Algorithm, and Networks, vol. 4, (2005), pp. 2551-2555.

[7] E. Kim, D. Kim and J. Park, "Min-Distance Hop Count based Multi-Hop Clustering In Non-uniform Wireless Sensor Networks", International Journal of Contents, vol. 8, no. 2, (2012) June, pp. 13-18.

[8] W. Heizelman, A. Chandrakasan and H. Balakrishnan, "An Application-Specific Protocol Architecture for Wireless Microsensor Networks", IEEE Transactions on Wireless Communications, vol. 1, no. 4, (2002) October, pp. 660-670.

[9] S. Saleh, A. Al-Awamry and F. M. Mahmoud, "Energy-Efficient Communication Protocol for Wireless Sensor Networks", International Journal of Engineering Research \&Technology, vol. 4, (2015) May.

[10] O. Younis and S. Fahmy, "HEED: A Hybrid Energy-Efficient Distributed Clustering Approach for Ad Hoc Sensor Networks", IEEE Transaction on Mobile Computing, vol. 3, no. 4, (2004).

[11] H. Jeon, K. Park, D.-J. Hwang and H. Choo, "Sink-oriented Dynamic Location Service Protocol for Mobile Sinks with an Energy Efficient Grid-Based Approach", MDPI Sensors, vol. 90, (2009) March, pp. 1433-1453.

[12] W. R. Heinzelman, A. P. Chandrakasan and H. Balakrishnan, "Energy-Efficient Communication Protocol for Wireless Microsensor Networks", Proc. of the Hawaii International Conference on System Sciences, (2000), pp. 3005-3014.

\section{Author}

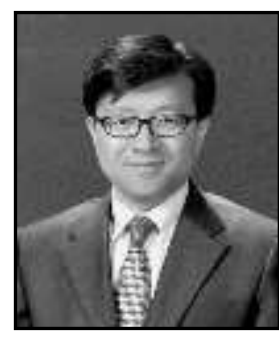

Joong-Ho Lee is currently a professor of Computer Science of the University of Yongin. He received his BS degree in Electronics \& Computer Engineering from University of Ulsan in 1988 and received MS degree in Electronics \& Computer Engineering from University of Ulsan in 1990. He received PhD degree in Electronics \& Computer Engineering from University of Ulsan in 1994. He worked as research engineer in SK-Hynix from 1994 to 2012. He was involved in DDR1, DDR2, and DDR4 SDRAM design. 